STIs, Ouagadougou, and `Dept of Pulmonary Care, "Sanou Souro" National Hospital, University of Bobo Dioulasso, Bobo Dioulasso, Burkina Faso. "Institute of Infectious and Tropical Diseases, Brescia University, Brescia, ${ }^{f}$ Emerging Bacterial Pathogens Unit, San Raffaele Scientific Institute, Milan, and **WHO Collaborating Centre for TB and Lung Diseases, Fondazione S. Maugeri, Care and Research Institute, Tradate, Italy.

Correspondence: A. Matteelli, Infectious and Tropical Diseases Institute, University of Brescia, Piazza Spedali Civili, 125100 Brescia, Italy. E-mail: matteelli@med.unibs.it

Support Statement: This study was performed in the framework of the agreement for technical support requested by the Burkina Faso National TB Programme to the University of Brescia, Italy and the TB Supranational Reference Laboratory (SRL) at the San Raffaele Institute, Milan, Italy. It was partially supported by Italian cooperation: WHO grant SRL-HSR, University of Brescia and Global Fund grant BUR 404-T.

Statement of Interest: None declared.

Acknowledgements: We thank K. Théophile and K. Mariam (both Dept of Pulmonary Care, "Yalgado" National Hospital, Ouagadougou, Burkina Faso).

\section{REFERENCES}

1 World Health Organisation (WHO). Multidrug and Extensively Drug-Resistant TB (M/XDR-TB) - 2010 Global Report on Surveillance and Response. WHO/HTM/TB/2010.3. Geneva, WHO, 2010.
2 World Health Organization (WHO). Global Tuberculosis Control 2008. Surveillance, Planning, Financing. Geneva, WHO, 2008.

3 World Health Organization (WHO). Guidelines for the Programmatic Management of Drug Resistant Tuberculosis. Geneva, WHO, 2008.

4 Raviglione MC, Uplekar MW. WHO's new Stop TB Strategy. Lancet 2006; 367: 952-955.

5 World Health Organisation (WHO). Treatment of Tuberculosis: Guidelines for National Programmes, 3rd Edn. WHO/CDS/TB/ 2003.313. Geneva, WHO, 2003.

6 National Tuberculosis Program (NTP), Ministry of Health Burkina Faso: Guide technique de lutte contre la tuberculose 2008 [Technical TB guide 2008. Edited by the National Tuberculosis Programme]. Ouagadougou, NTP, 2008.

7 Rusch-Gerdes S, Pfyffer GE, Casal M, et al. Multicentre laboratory validation of the Bactec MGIT 960 technique for testing susceptibilities of Mycobacterium tuberculosis to classical second line drugs and newer antmicrobials. J Clin Microbiol 2006; 44: 688-692.

8 Miotto P, Saleri N, Dembelé M, et al. Molecular detection of rifampin and isoniazid resistance to guide chronic TB patient management in Burkina Faso. BMC Infect Dis 2009; 9: 142.

9 Chen-Yuan C, Enarson DA, Fujiwara PI, et al. Strategies of extensively drug resistance TB risk management for health workers and other care givers. Expert Rev Respir Med 2008; 2: 47-54.

10 Sotgiu G, Ferrara G, Matteelli A, et al. B. Epidemiology and clinical management of XDR-TB: a systematic review by TBNET. Eur Respir J 2009; 33: 871-881.

11 Migliori GB, Besozzi G, Girardi E, et al. Clinical and operational value of the extensively drug-resistant tuberculosis definition. Eur Respir J 2007; 30: 623-626.

12 Buijtels PCAM, van der Sande MAB, de Graaff CS, et al. Nontuberculous mycobacteria, Zambia. Emerg Infect Dis 2009; 15: 242-248.

\title{
Impaired pulmonary function and the risk of tuberculosis: a population-based cohort study
}

\section{To the Editors:}

It is well known that pulmonary tuberculosis (TB) can cause lung impairment leading to chronic obstructive pulmonary disease (COPD) [1], but less is known to what extent impaired lung function increases the risk of TB. A case-control study from the UK found patients with emphysema to have a threefold increased risk of $\mathrm{TB}$, adjusted for smoking and the use of corticosteroids [2]. A cohort study from Denmark found moderate-to-severe COPD to be associated with a two- to three-fold increased risk of hospitalisation with TB [3]. We have previously shown that patients hospitalised with COPD have a three-fold increased risk of active TB compared with population controls [4]. In the present study, we examine the risk of active TB in relation to lung function in a cohort who underwent spirometry in 1974-1992.

Between 1974 and 1992, 22,444 males and 10,902 females, born 1921-1949, participated in a health screening programme, the Malmö Preventive Project. Complete birth cohorts, born in pre-specified years, from the city of Malmö were invited; the overall participation proportion was $\sim 70 \%$ [5]. The screening included physical examination, spirometry, blood samples and assessment of lifestyle factors by means of a self-administered questionnaire. Some questions varied between the cohorts. We excluded 4,413 individuals because of missing spirometry data; 26 individuals were excluded because of missing data on either body mass index (BMI), smoking or immigration status, leaving a total of 28,907 individuals in final analysis $(21,174$ males and 7,733 females).

BMI as was calculated as mass $/$ height $^{2}\left(\right.$ in $\mathrm{kg} \cdot \mathrm{m}^{-2}$ ). Individuals were regarded as having diabetes mellitus if they answered positively to the question "Do you have diabetes mellitus?" or their fasting blood glucose level was $\geqslant 6.1 \mathrm{mmol} \cdot \mathrm{L}^{-1}$.

Around $96 \%$ of the cohort $(n=27,789)$ answered questions on alcohol. Individuals were regarded as having "problematic drinking" if they answered affirmatively to four or more questions on drinking habits and were regarded as having 
chronic bronchitis if reporting episodes of chronic productive cough lasting $>3$ months for more than two consecutive years. Individuals were categorised as current smokers if they answered affirmatively to the question "Are you a smoker?"

$57 \%$ of the cohort was presented a question regarding precedent TB: "Have you had TB?"

Assessment of hospitalisations prior to screening and during follow up, immigration status, socioeconomic status and incident cancer were assessed by record linkage between national databases via a unique, lifelong personal identity number assigned to each person living in Sweden.

Forced vital capacity and forced expiratory volume in $1 \mathrm{~s}$ (FEV1) were measured by spirometry at the screening examination; FEV1 values were analysed as \% predicted. The predicted values were obtained using equations derived from linear regression of 3,467 male and 2,961 female never-smokers in the present cohort and classified according to Global Initiative for Obstructive Lung Disease (GOLD) staging, as described previously [6, 7].

The Regional Center of Communicable Disease Control (Malmö, Sweden) has held a TB register since 1989. Active TB is notifiable by law in Sweden and reporting is done in parallel by the microbiological laboratories and clinicians. Outcome was defined as the first episode of TB notified in the local TB register. Follow-up ended with the first episode of TB, date of emigration, date of death or December 31, 2008, whichever came first.

Chi-squared tests and one-way ANOVA were used to assess the distribution of risk factors for TB between groups with different levels of lung function. Cox proportional hazards models were used to estimate hazard ratios (HRs) of subsequent
TB. FEV1 \% pred was modelled as a continuous variable; GOLD stage was modelled as categorical. Potential confounders were selected a priori: smoking status, screening age, sex, bronchitis, immigration status, socioeconomic status, BMI, diabetes mellitus, previous $\mathrm{TB}$, alcohol consumption, cancer, HIV and silicosis were considered. Effect modification by smoking, immigration status, sex and birth cohort was assessed by examining incidence rates of $\mathrm{TB}$ in different strata of the covariates and by entering an interaction term to the fully adjusted Cox models.

Proportionalities of hazards were assessed graphically and by testing for a non-zero slope in a generalised linear regression of the scaled Schoenfeld residuals as a function of time. All analyses were performed using STATA SE (version 10.1 for Windows; StataCorp LP, College Station, TX, USA).

A total of 26 TB cases (5.2 per 100,000 person-yrs, 95\% CI 3.67.7) were notified in the TB register during the follow-up in 1989-2008. TB incidence was inversely correlated with FEV1\% pred (HR per $10 \%$-unit increase $0.71,95 \%$ CI $0.59-0.86$ ). The FEV1-TB association persisted after adjustment for current smoking and age at screening (HR per $10 \%$-unit increase 0.75 , 95\% CI 0.61-0.91). Although the stratified analyses were based on small numbers, the results were largely consistent in different strata of the covariates and all p-values from likelihood ratio tests for interaction were $>0.05$.

24 cases of TB occurred among 25,869 participants, classified according to the GOLD criteria. The incidence of TB increased with GOLD stage (stage I crude HR 1.9, 95\% CI 0.5-6.7; stage II HR 5.64, 95\% CI 2.2-14.7; and stage III-IV HR 6.9, 95\% CI $0.9-$ 52.6; $\mathrm{p}<0.001$ for linear trend), although only one case of $\mathrm{TB}$ was GOLD stage III-IV.

TABLE 1 Relationships between risk factors and incidence of tuberculosis (TB) in the Malmö Preventive Project ${ }^{\#, \text {. }}$

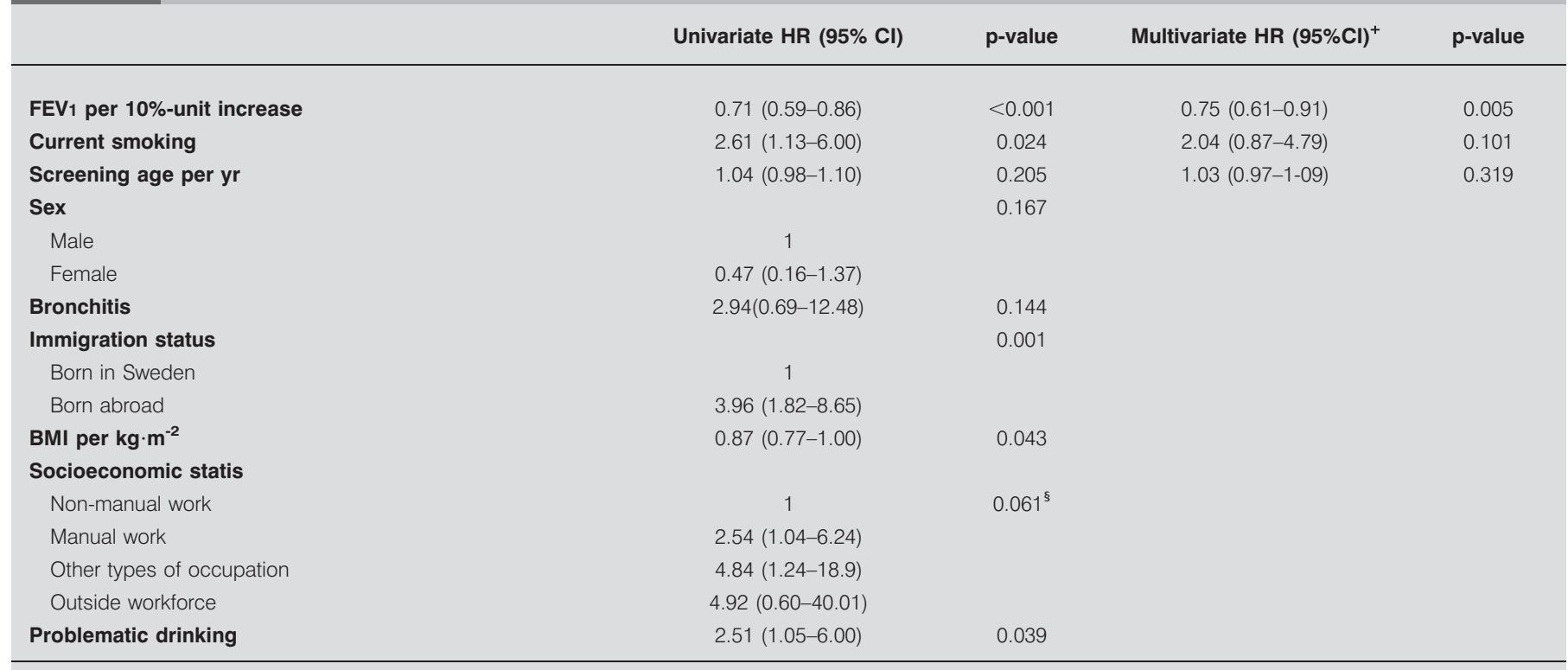

HR: hazard ratio; FEV1: forced expiratory volume in $1 \mathrm{~s}$; BMI: body mass index. ${ }^{*}$ : FEV 1 , screening age and smoking were forced into the model; no other risk factor confounded the FEV1-TB association (FEV1 estimates changed 0.3-2.3\%) and were therefore not included; ": estimated by Cox regression using time since entry as time scale; ${ }^{+}$: adjusted for all the parameters listed; ${ }^{\text {s. }}$ global p-value from likelihood ratio test. 
The limited number of TB cases restricted the number of covariates in the final multivariate model. Smoking and screening age were included by forced entry. Additional confounders were examined separately in univariate Cox models (table 1) and in Cox models including FEV1, screening age and smoking. None of the additional covariates (BMI, immigration status, problematic drinking, bronchitis, sex and socioeconomic status) confounded the FEV1-TB association in the models with FEV1, screening age and smoking (FEV1 estimates changed $0.3-2.3 \%$ ), and were therefore not included in the final model (table 1). None of the participants had a hospital discharge diagnosis listing silicosis or HIV prior to screening. During follow-up, there were 24 cases of HIV and five cases of silicosis. None of these developed TB, nor did any of the 1,109 participants with diabetes at baseline. Seven individuals developed both cancer and active TB during follow-up, but only one of the episodes of cancer occurred within a year from the TB diagnosis.

None of the 81 participants who had a hospital discharge listing TB or TB sequelae (International Classification of Diseases (ICD) 8: 011-019; ICD 9: 010-018 and 131; ICD 10: A15-A19 and B90) in the in-patient register prior to screening developed TB during follow-up. A stratified analysis in the subgroup who stated no history of $\mathrm{TB}$ in the baseline questionnaire $(n=16,291)$ was performed: 10 cases of TB occurred during follow-up (crude HR per 10\%-unit increase in FEV1 0.68, 95\% CI 0.50-0.92).

Data on both current and former smoking status were available in $85 \%$ of the participants. Rerunning the analyses in this subcohort $(n=25,804)$ adjusting for smoking status (current, former or never) and screening age yielded similar results (HR for $10 \%$-unit increase in FEV1 \% pred $0.74,95 \%$ CI $0.61-0.91$ ).

In this population-based cohort study from a low TB-incidence region, we show that decreased lung function is associated with an increased risk of active TB.

Mycobacterium tuberculosis infects phagocytic cells in the lung; the clearance rate is not known, but infection is in many cases chronic. Antigen presentation by infected macrophages and subsequent induction of a T-cell response is crucial for controlling infection [8].

In COPD, innate defence mechanisms in the airways are impaired, mainly by decreased mucociliary clearance, but additional mechanisms (e.g. decreased macrophage function) have been described $[9,10]$. Factors conferring vulnerability to respiratory infection in COPD might also play a role in increasing the risk of TB infection and/or progression to active TB disease. However, it should be noted that the relationships between FEV1 and TB were seen over the entire range of FEV1 in this population study, and was not limited to subjects with COPD.

A limitation of the present study is the small number of TB cases. Another limitation is the lack of data on previous TB for a large proportion of the cohort. Residual confounding from other factors, such as medication with corticosteroids, cannot be ruled out. Due to the epidemiological situation in Sweden, we believe that most cases of TB in this study are reactivations from latent $\mathrm{TB}$; however, it was not possible to identify individuals with latent TB infection at baseline. The spirometry in the health screening did not meet modern standards. However, previous studies from this cohort have shown that the validity is acceptable in the context of an epidemiological study [6].

Despite these limitations, there seems to be growing evidence that impaired lung function and COPD are associated with increased incidence of active TB. Further research is needed, especially in high TB-incidence regions, to disentangle the underlying contributory factors and to judge whether individuals with impairment of lung function are to be viewed as a risk group warranting targetted TB prevention measures.

\section{Inghammar, ${ }^{*}$, C-G. Löfdahl ${ }^{\#}$, N. Winqvist ${ }^{\oplus}$, B. Ljungberg*, A. Egesten ${ }^{\#}$ and G. Engström ${ }^{+}$} *Section for Infection Medicine, "Section for Respiratory Medicine, Dept of Clinical Sciences Lund, Lund University, Skåne University Hospital, Lund, "Infectious Disease Research Unit, and ${ }^{+}$Cardiovascular Epidemiology Research Group, Dept of Clinical Sciences Malmö, Lund University, Skåne University Hospital, Malmö, Sweden.

Correspondence: M. Inghammar, Dept of Infectious Diseases, Skåne University Hospital, SE-221 85 Lund, Sweden. E-mail: Malin.Inghammar@med.lu.se

Statement of Interest: A statement of interest for G. Engström can be found at www.erj.ersjournals.com/site/misc/statements. xhtml

\section{REFERENCES}

1 Salvi SS, Barnes PJ. Chronic obstructive pulmonary disease in nonsmokers. Lancet 2009; 374: 733-743.

2 Jick SS, Lieberman ES, Rahman MU, et al. Glucocorticoid use, other associated factors, and the risk of tuberculosis. Arthritis Rheum 2006; 55: 19-26.

3 Benfield $\mathrm{T}$, Lange $\mathrm{P}$, Vestbo J. COPD stage and risk of hospitalization for infectious disease. Chest 2008; 134: 46-53.

4 Inghammar M, Ekbom A, Engström G, et al. COPD and risk of tuberculosis - a population based cohort study. PLoS One 2010; 5 e10138.

5 Berglund G, Nilsson P, Eriksson KF, et al. Long-term outcome of the Malmo preventive project: mortality and cardiovascular morbidity. J Intern Med 2000; 247: 19-29.

6 Ekberg-Aronsson M, Pehrsson K, Nilsson JA, et al. Mortality in GOLD stages of COPD and its dependence on symptoms of chronic bronchitis. Respir Res 2005; 6: 98.

7 Pauwels RA, Buist AS, Calverley PM, et al. Global strategy for the diagnosis, management, and prevention of chronic obstructive pulmonary disease. NHLBI/WHO Global Initiative for Chronic Obstructive Lung Disease (GOLD) Workshop summary. Am J Respir Crit Care Med 2001; 163: 1256-1276.

8 Tufariello JM, Chan J, Flynn JL. Latent tuberculosis: mechanisms of host and bacillus that contribute to persistent infection. Lancet Infect Dis 2003; 3: 578-590.

9 Sethi S, Murphy TF. Infection in the pathogenesis and course of chronic obstructive pulmonary disease. N Engl J Med 2008; 359: 2355-2365.

10 Taylor AE, Finney-Hayward TK, Quint JK, et al. Defective macrophage phagocytosis of bacteria in COPD. Eur Respir J 2010; 35: 1039-1047. 